\title{
Lanthanum Telluride: Mechanochemical Synthesis of a Refractory Thermoelectric Material
}

\author{
Andrew May ${ }^{1}$, Jeff Snyder ${ }^{1}$, and Jean-Pierre Fleurial ${ }^{2}$ \\ ${ }^{1}$ California Institute of Technology, 1200 E. California Blvd. Pasadena CA, 91106 \\ ${ }^{2}$ Jet Propulsion Laboratory, California Institute of Technology, 4800 Oak Grove Dr., Pasadena, CA 91109 \\ ${ }^{2}$ (818) 354-4144, jean-pierre.fleurial@jpl.nasa.gov
}

\begin{abstract}
Recent experimental work on lanthanum telluride has confirmed its significant potential as an n-type material for high temperature thermoelectric (TE) power generation application. The phase of interest, $\mathrm{La}_{3-\mathrm{x}} \mathrm{Te}_{4}$, has a $\mathrm{Th}_{3} \mathrm{P}_{4}$ defect structure where $x$ is the lanthanum vacancy with values ranging between 0 and $1 / 3$. Thermoelectric properties change rapidly with $x$ since the carrier concentration, $n$, is proportional to the (1-3x) parameter. Controlling the Te to La stoichiometry in lanthanum telluride is thus vital to achieving the optimum self-doping level for the highest dimensionless figure of merit ZT value. We report on a significant improvement in reproducibly preparing this refractory compound over prior lengthy and unwieldy high temperature experimental techniques developed in the 1980 's. Mechanochemical processes are utilized to synthesize precise stoichiometries of lanthanum telluride at room temperature, enabling improved characterization, analysis and modeling of its transport properties as a function of the number of La vacancies. We report TE properties for a large range of the allowed compositions, with ZT values greater than 1.0 obtained at $1275 \mathrm{~K}$ for several compositions. In addition to stoichiometric optimization within the pure compound, chemical substitutions can enhance performance by decreasing the lattice thermal conductivity and tuning the electrical properties for maximum ZT values at lower temperatures; preliminary studies indicate that the addition of ytterbium increases ZT. Some properties pertaining to device development are discussed. Specifically, lanthanum telluride has a low sublimation rate, and a coefficient of thermal expansion that closely matches a p-type rare earth compound analog (the $\mathrm{Yb}_{14} \mathrm{MnSb}_{11}$ Zintl compound).
\end{abstract}

Keywords: thermoelectric, mechanochemical, mechanical alloying, refractory materials.

PACS: 84.60.Rb; 85.80.Fi; 89.30.Gg

\section{INTRODUCTION}

The need for high-temperature materials for TE power generation led to investigations of lanthanum telluride, and other rare-earth chalcogenides of the thorium phosphide $\left(\mathrm{Th}_{3} \mathrm{P}_{4}\right)$ structure type, at the Jet Propulsion Laboratory and the Thermo Electron Corporation during the late 1980s (Wood, 1988). These studies demonstrated that lanthanum telluride had significant promise for deep-space power generation due to its thermal stability and large thermoelectric dimensionless Figure of Merit, ZT, which is a material property that directly impacts device efficiency. It was also shown that the transport properties of lanthanum telluride are very sensitive to oxygen content and sample composition. The chemical formula is written as $\mathrm{La}_{3-x} \mathrm{Te}_{4}$, where $x$ represents a lanthanum vacancy and is restricted to $0 \leq x \leq 1 / 3$. The electronic behavior of the system is easily understood in terms of atomic valence. Lanthanum and tellurium have +3 and -2 oxidation states, respectively, and thus one would expect a charge-balanced $\mathrm{La}_{2} \mathrm{Te}_{3}$ compound to form. However, this is not the case, and $\mathrm{La}_{2} \mathrm{Te}_{3}$ is only realized when $x=1 / 3$, which is when one-ninth of the lanthanum atoms are removed from the filled structure. When $x<1 / 3$, free electrons are introduced into the conduction band, and thus lanthanum telluride and similar rare-earth chalcogenides are natural n-type conductors. The chemical environment within the crystal can be described by $\left(\mathrm{La}^{+3}\right)_{3-\mathrm{x}} \mathrm{V}_{\mathrm{x}}\left(\mathrm{Te}^{-2}\right)_{4}\left(\mathrm{e}^{-1}\right)_{1}$. 3x, where $V$ is a lanthanum vacancy and $e$ is a free electron. This notation demonstrates that when $x=0$ each formula unit donates one free electron to the system, and when $x=1 / 3$ there are no free electrons. Therefore, a natural metal-insulator transition exists and the concentration of free electrons in the system can be determined by 
$n=n_{\max }(1-3 x)$, where $\mathrm{n}_{\max }$ is the number of free electrons when $x=0\left(\sim 4.5 \times 10^{21} \mathrm{~cm}^{-3}\right)$. This electronic behavior makes the system particularly interesting for scientific study and thermoelectric optimization. However, because the lanthanum concentration varies by less than three atomic percent while the carrier concentration covers 21 orders of magnitude, performing such tasks requires excellent control over stoichiometry.

To perform a thorough investigation of this system it is necessary to have a synthesis technique that allows for homogeneous samples of desired compositions to be produced. The techniques originally utilized to study these rare-earth chalcogenides were high temperature low yield processes, involving solid-state diffusion and melting (Danielson, 1988; Vining, 1988; Cutler, 1964). Lanthanum telluride melts at approximately 1775K (Ramsey et al., 1965) which makes melt processing more difficult. In addition to taking at least one week to complete, these high temperature processes are extremely sensitive to oxidation and can also result in the loss of tellurium via sublimation. Therefore, $\mathbf{n}$-type samples produced in the late 1980s did not achieve a high level of reproducibility due to the presence of second phases and/or unsatisfactory correlation between nominal and actual Te/La stoichiometry. After completing the evaluation of the n-type compositions, a lot of effort was then devoted to producing p-type compositions of the rare-earth chalcogenides, which proved to be a very difficult challenge. As a result, research supported by NASA on high temperature thermoelectric materials reverted in the 1990's to developing incremental improvements in the performance of proven n-type and p-type $\mathrm{Si}_{1-\mathrm{x}} \mathrm{Ge}_{\mathrm{x}}$ alloys.

Renewed interest in achieving significant increases in thermoelectric energy conversion for power generation from high temperature heat sources has led JPL to revisit refractory rare earth compounds. Recent results on a p-type rareearth compound, the $\mathrm{Yb}_{14} \mathrm{MnSb}_{11}$ Zintl phase, with $\mathrm{ZT}$ values exceeding 1.0 at $1273 \mathrm{~K}$, have been reported (Brown, 2006; Caillat, 2007). We report on parallel efforts focused on developing a more practical and scalable technique for preparing and characterizing $\mathrm{La}_{3-\mathrm{x}} \mathrm{Te}_{4}$ and related rare earth chalcogenides. A mechanochemical process has been utilized to form the desired phase at room temperature, which greatly reduces the risk of oxidation and tellurium loss. This technique allows for the rapid production of a variety of compositions, while maintaining reasonable control over the Te/La stoichiometry produced.

\section{SYNTHESIS AND CHEMICAL ANALYSIS}

Mechanical alloying transforms elemental lanthanum and tellurium into the desired $\mathrm{Th}_{3} \mathrm{P}_{4}$ structure at ambient temperatures. Elemental species are obtained from Alfa Aesar; lanthanum and tellurium chunks with metals basis purity $99.9 \%$ and $99.999 \%$, respectively, are utilized. Lanthanum and tellurium are slivered in inert atmosphere, and combined in a milling vial which is placed into a high-energy ball mill. The milling is completed in less than a day, and yields are greater than $90 \%$.

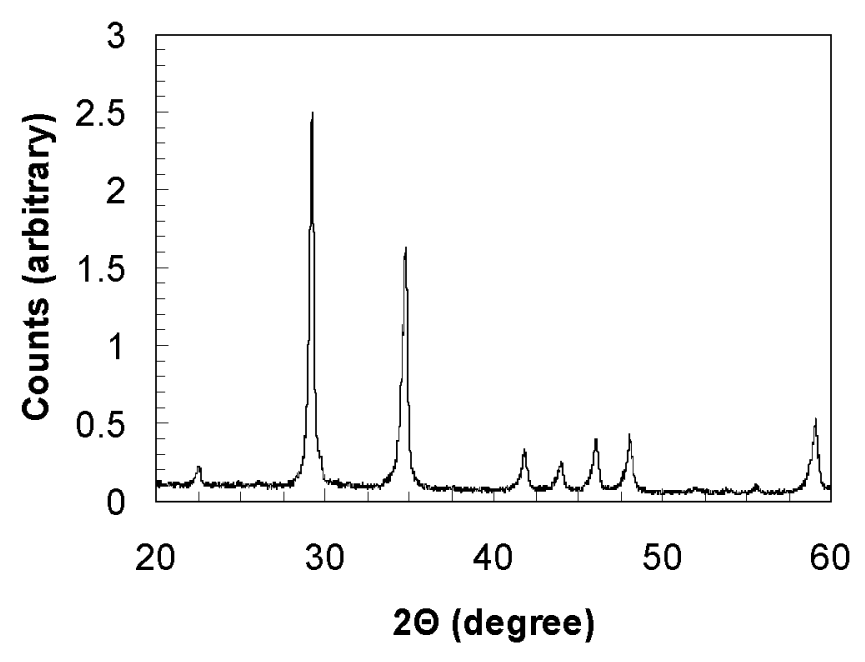

(a) X-ray Diffraction Pattern.

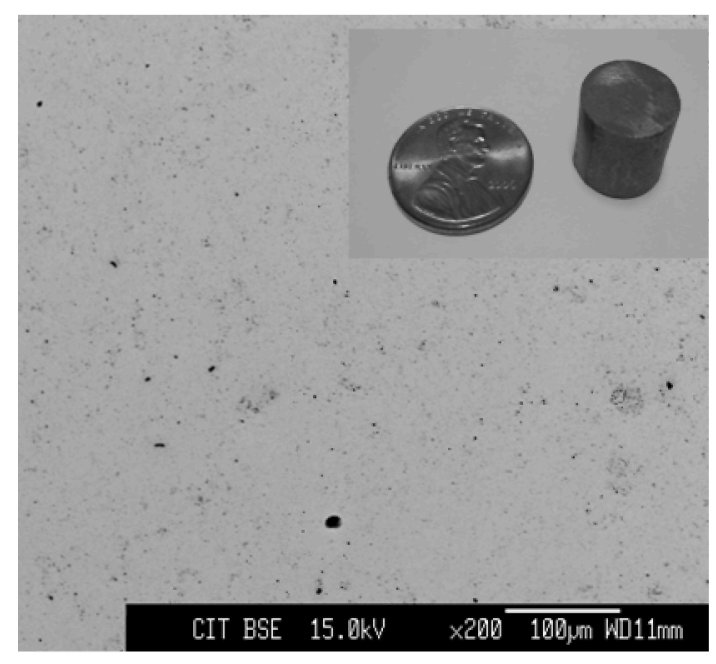

(b) Electron Microprobe Image.

FIGURE 1. Chemical and phase analysis of $\mathrm{La}_{2.82} \mathrm{Te}_{4}$ : The $\mathrm{X}$-ray diffraction pattern shows only the $\mathrm{Th}_{3} \mathrm{P}_{4}$ phase is present. The electron microprobe image demonstrates sample compositional uniformity and near theoretical density. 
An argon/vacuum hot-press is utilized to sinter the powder at $1350-1450 \mathrm{~K}$. The typical sample size is approximately $1 \mathrm{~cm}$ in length and $1.2 \mathrm{~cm}$ in diameter, as shown in the insert of Figure $1 \mathrm{~b}$ where a penny is provided for scale; samples greater than $2 \mathrm{~cm}$ in length have been produced. Sample density is generally in excess of $95 \%$ of theoretical, with exceptions noted for tellurium rich samples that have proved more difficult to sinter. Samples are typically sintered powder from a single milling vial. However, combining consecutive milling batches is one route to obtain several samples of the same composition. When this is performed, small samples of each powder batch are pressed to determine that the powders are near the desired composition before combining with the main powder source.

After synthesis, the compounds are examined by X-ray diffraction (XRD) and electron microprobe for phase purity. The XRD patterns are examined (see Figure 1a), and expected impurities, such as $\mathrm{La}_{2} \mathrm{O}_{2} \mathrm{Te}, \mathrm{LaTe}_{2}$ and $\mathrm{LaTe}_{3}$ are readily identified; in Figure 1a only $\mathrm{La}_{3-\mathrm{x}} \mathrm{Te}_{4}$ peaks are observed. Most samples are homogeneous and free of any second phase; the XRD patterns for some samples have indicated trace amounts of oxidation. The electron microprobe image (Figure 1b) was generated with back-scattered electrons to create a compositional contrast image, where regions of different tone reveal different compositions. The sample is found to be single phase; close examination shows that all dark regions are attributed to pores. The electron microprobe is equipped with a wavelength dispersive spectrometer (WDS) to analyze sample composition for specified elements. The sample shown in Figure $1 \mathrm{~b}$ was produced by combining three separate batches of $\mathrm{La}_{2.82} \mathrm{Te}_{4}$ powder, and therefore the distribution of compositions is representative of the consistency across three samples. In this sample, six points of roughly one square micron were examined, and the mean atomic percent lanthanum was found to be 42.04 , compared to the nominal composition of 41.32 at. \% La. The standard deviation and range were 0.19 and 1.24 atomic percent La, respectively. In the typical sample produced from one powder source, the standard deviation and range are 0.11 and 0.34 at.\% La, respectively, with ranges as small as 0.08 at.\% La observed. We have determined there is a systematic error which increases the relative amount of lanthanum to tellurium in the WDS calculation. We believe this is a due to the use of lanthanum phosphate and elemental tellurium for standards combined with a ZAF correction method that was not developed for rare-earth chalcogenides.

\section{THERMOELECTRIC PROPERTIES}

The pertinent electronic properties are the electrical resistivity, $\rho$, and Seebeck coefficient, $\alpha$. The Seebeck coefficients of all compositions are negative, indicating the desired n-type conduction. Magnitudes of the Seebeck coefficient and electrical resistivity increase with decreasing carrier concentration, or increasing $x$, and with increasing temperature. The results from the current study are consistent with the previous work, as shown in Figure $2 \mathrm{a}, \mathrm{b}$, where $\rho$ and $\alpha$ at $1273 \mathrm{~K}$ are plotted versus nominal $x$ values for both studies (Danielson, 1988). In Figure 2 the solid markers represent this study and the open markers represent the previous work. Also included in Figure 2 are solid-curves generated using a semi-empirical model developed to describe the data presented for the previous work (Vining, 1988).

When examining Figure 2, the experimental error in producing a given composition must be considered, as well as the error within the measurement itself. The errors associated with these two processes are difficult to obtain because the error in $x$ creates an apparent error in $\rho$ and $\alpha$. By utilizing the WDS standard deviation for typical samples, the corresponding error in $x$ is approximately 0.01 (recall $0 \leq \mathrm{x} \leq 1 / 3$ ). We believe there are approximately $5 \%$ error in electrical resistivity and perhaps a $10 \%$ error in Seebeck coefficient. The error in $x$ is enough to account for the deviation between the two sets of data.

The ability to produce a desired composition has been stated as critical. In fact, it is the ability to reproducibly create samples with desired properties that is critical. In this way, the properties themselves can be viewed of as a compositional map for a given synthesis technique. The figure below demonstrates that the composition maps of the two synthesis techniques discussed are similar, but we do not expect them to be the same. For instance, the ball milling samples appear to be more resistive, which can be explained in two ways: (a) the milling creates finer grains which causes increased charge carrier scattering, or (b) the high temperature synthesis leads to tellurium losses resulting in lower values of $x$ than expected from nominal composition. In either case, it is necessary to show that properties can be reproduced between samples. This can be done in a few different ways. One way is to produce small batches and compare the deviation between them. This is shown in Figure 3, where the electrical resistivity as a function of temperature for two samples with $x=0.08$ is provided. The electrical properties of these two samples 
are very consistent, and it is almost difficult to differentiate the two; one is shown in grey and the other as open black circles.

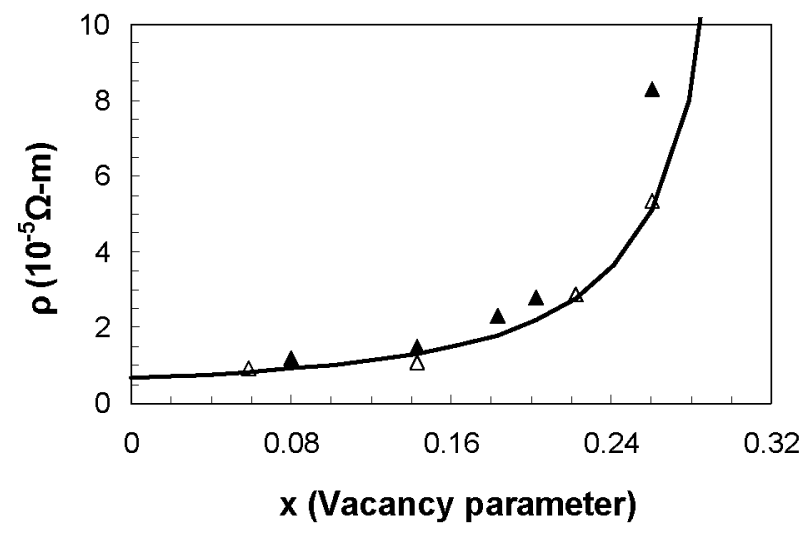

(a) Electrical Resistivity versus $x$.

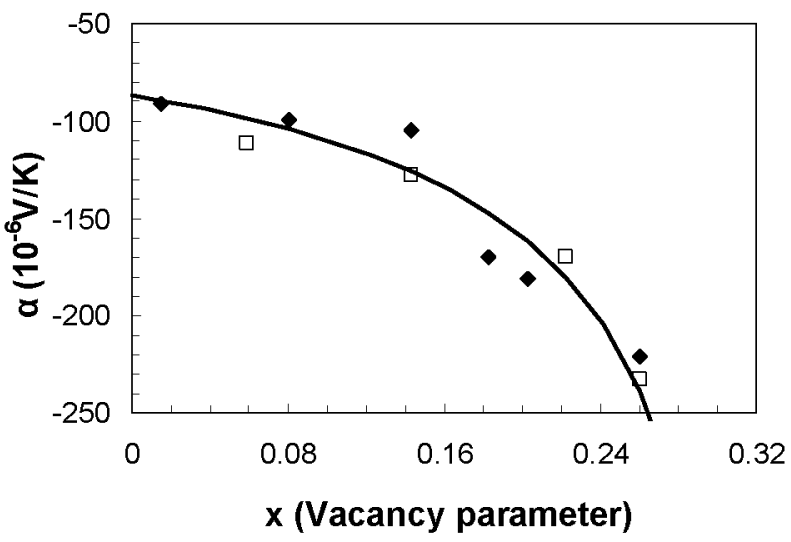

(b) Seebeck Coefficient versus $x$.

FIGURE 2. Dependence of thermoelectric properties on composition at $1273 \mathrm{~K}$; the current study is shown by filled markers, previous work is shown as open markers, and the solid curves are from a semi-empirical model by Danielson and Vining.

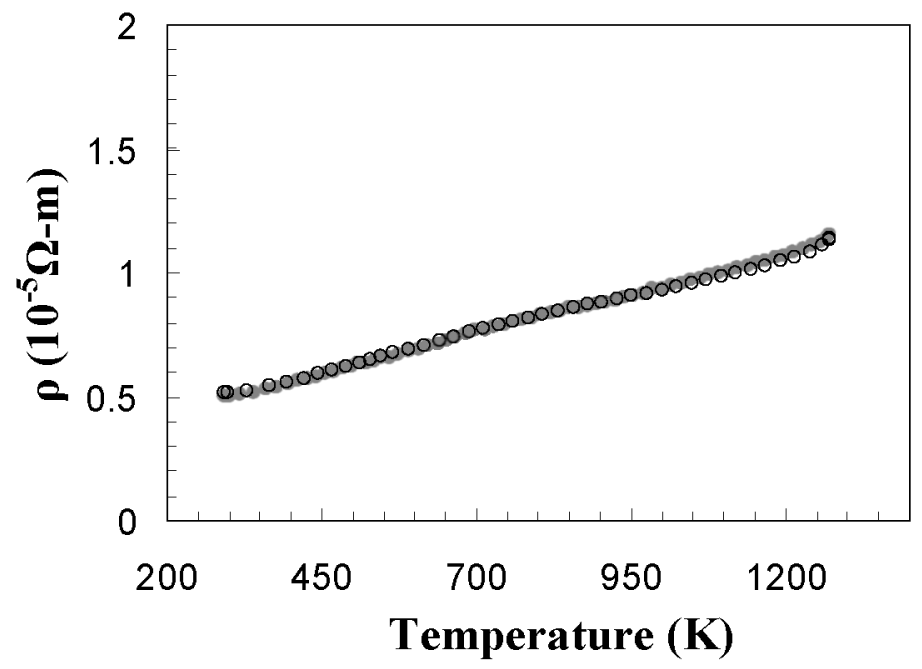

FIGURE 3. The electrical resistivity for two samples with nominal composition $\mathrm{La}_{2.92} \mathrm{Te}_{4}$ is consistent for all temperatures.

Another way to demonstrate samples with consistent properties can be made is to combine the small powder batches into a large batch, and produce samples from the combined powder. This was completed by combining the powder from three milling vials and pressing three pellets, a small number was chosen to prove the concept without requiring an excessive time commitment. The pellets were sliced and the room temperature Seebeck coefficient and electrical resistivity was measured on the various slices. The Seebeck coefficient of these slices is plotted versus the corresponding electrical resistivity in Figure 4a, where the samples (different pressed pellets) are differentiated by use of dark circles, open circles, and grey circles. The variation between samples, and within a sample, is found to be acceptable. Three pieces were selected for high temperature thermal diffusivity measurement, two of which were from the same sample. The thermal diffusivity, shown for the three slices in Figure $4 \mathrm{~b}$, is combined with heat capacity and density to yield thermal conductivity, $\kappa$, a key TE property. The deviation between slices is observed by comparing the closed squares and open circles to the grey triangles, and the deviation within a slice is that between the open circles and closed squares. In this case, the greatest deviation between slices is approximately $10 \%$, a value that is acceptable when attempting to produce samples with large, consistent $\mathrm{ZT}_{\text {in }}$ the $\mathrm{La}_{3-\mathrm{x}} \mathrm{Te}_{4}$ system. 


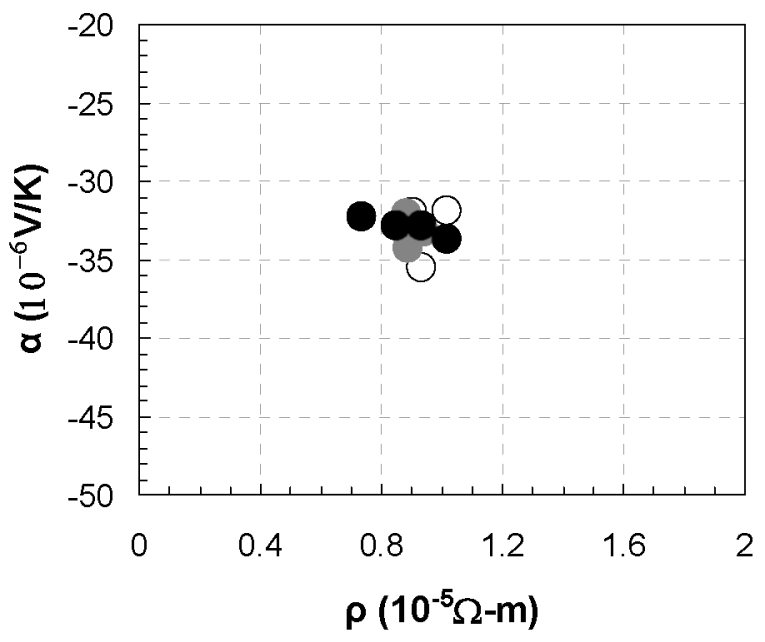

(a) Seebeck coefficient versus electrical resistivity.

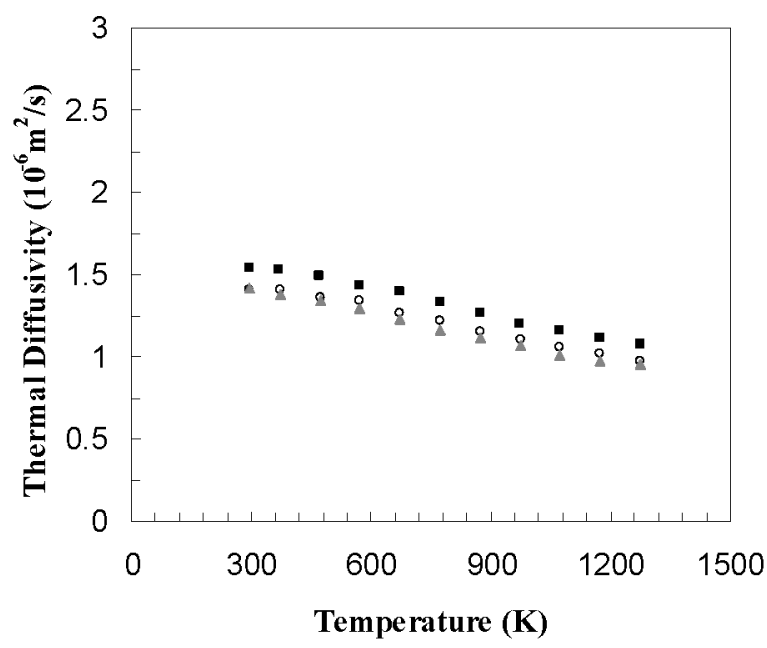

(b) Thermal Diffusivity versus temperature.

FIGURE 4. The TE properties of three samples produced by combining powder from three separate milling batches are shown to demonstrate the variation of properties within such an experiment.

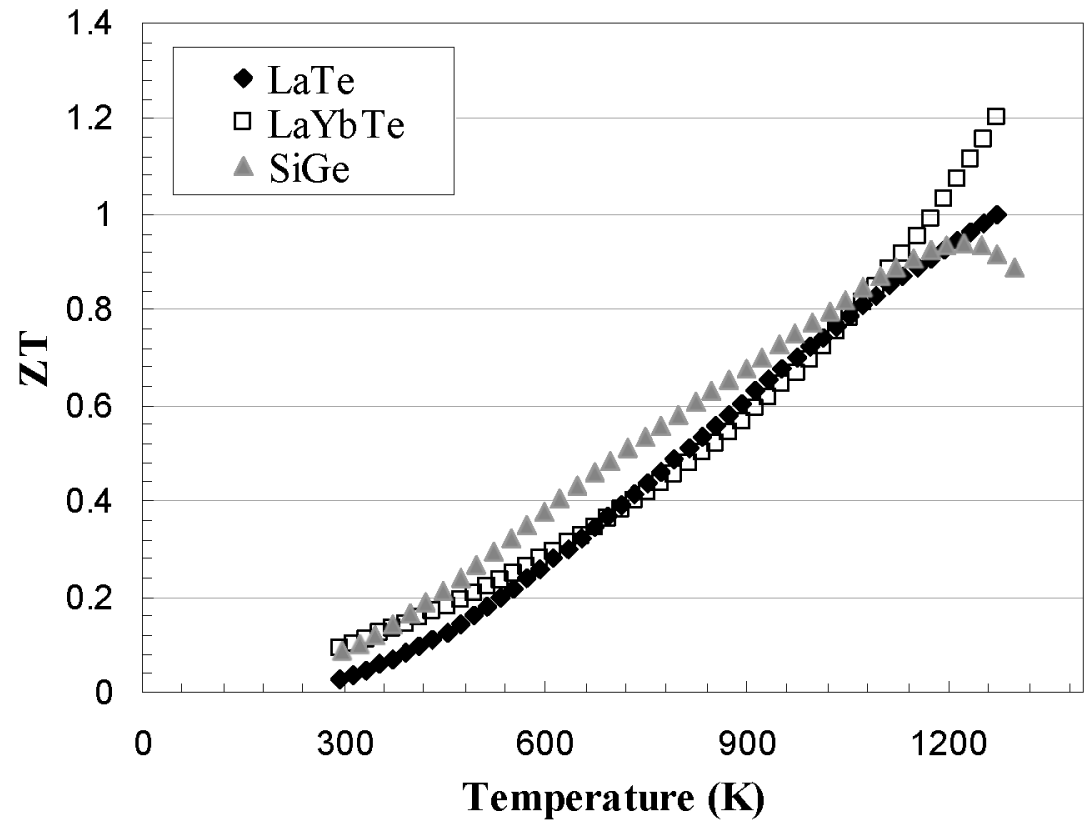

FIGURE 5. A comparison of the temperature dependence of ZT for n- $\mathrm{Si}_{0.8} \mathrm{Ge}_{0.2}, \mathrm{La}_{3-\mathrm{x}} \mathrm{Te}_{4}$ and $\mathrm{La}_{3-\mathrm{x}} \mathrm{Yb}_{\mathrm{y}} \mathrm{Te}_{4}$.

The lanthanum telluride system is attractive because the self-doping provided by lanthanum vacancies allows for the carrier concentration to be optimized for thermoelectric performance. We have found ZT values of approximately one at $1275 \mathrm{~K}$ for the pure $\mathrm{La}_{3-\mathrm{x}} \mathrm{Te}_{4}$ system for $x$ values near 0.2 . The system is also appealing because it is capable of accepting various chemical substitutions that may enhance TE performance. An investigation into the addition of $\mathrm{Yb}, \mathrm{Bi}$, and $\mathrm{Sb}$ to lanthanum telluride has begun. Preliminary ZT results are compared to the standard $\mathrm{n}-\mathrm{Si}_{0.8} \mathrm{Ge}_{0.2}$ in Figure 5. Data is presented for a pure lanthanum telluride sample as well as a sample containing $\mathrm{Yb}$. At this time, it appears the addition of $\mathrm{Yb}$ is capable of increasing the ZT from 1.0 to 1.2 at $1275 \mathrm{~K}$. Both samples have ZT greater than that of $\mathrm{n}$-type $\mathrm{n}-\mathrm{Si}_{0.8} \mathrm{Ge}_{0.2}$ used in space power systems. 


\section{THERMAL AND MECHANICAL PROPERTIES}

The desired thermoelectric properties must be combined with structural and chemical integrity at high temperatures for a material to be utilized in TE power generators. This is especially true for deep-space application, where the materials must be thermally stable at high temperatures (hot side temperatures near $1275 \mathrm{~K}$ ) for over 15 years. Preliminary data obtained on the thermophysical properties of lanthanum telluride are quite encouraging since the sublimation rate of $\mathrm{La}_{3-\mathrm{x}} \mathrm{Te}_{4}$ is at least as low as that of alloys. The beginning of life sublimation rates of $\mathbf{n}$ $\mathrm{Si}_{08} \mathrm{Ge}_{0.2}$, lanthanum telluride, and the high temperature p-type $\mathrm{Yb}_{14} \mathrm{MnSb}_{11}$ Zintl are compared in Table 1. The target long-term sublimation rate is $5 \times 10^{-7} \mathrm{~g} / \mathrm{cm}^{2} / \mathrm{hr}$, which is achieved using thin coatings of $\mathrm{Si}_{3} \mathrm{~N}_{4} / \mathrm{SiO}_{2}$ on $\mathrm{Si}_{0.8} \mathrm{Ge}_{0.2}$ (Wood, 1985).

Thermal expansion is another key property for device design. Matching coefficients of thermal expansion for the pand n-type legs greatly facilitates their integration into a thermocouple. In the late 1980's boron carbide was the candidate p-type high temperature material, a clear mismatch in terms of thermal and mechanical properties with ntype $\mathrm{La}_{3-\mathrm{x}} \mathrm{Te}_{4}$. From preliminary high temperature measurements, it is observed that the thermal expansion of $\mathrm{Yb}_{14} \mathrm{MnSb}_{11}$ and $\mathrm{La}_{3-\mathrm{x}} \mathrm{Te}_{4}$ match very well, while the coefficient of thermal expansion of $\mathrm{Si}_{0.8} \mathrm{Ge}_{0.2}$ is only about $25 \%$ that of the Zintl compound. The properties shown in Table 1, combined with the ZT demonstrated in Figure 5, provide strong support for the combined use of lanthanum telluride and $\mathrm{Yb}_{14} \mathrm{MnSb}_{11}$ in a high temperature thermoelectric couple.

TABLE 1. Comparison of beginning of life (BOL) sublimation rates and coefficient of thermal expansion at $1273 \mathrm{~K}$ for $\mathrm{La}_{3-\mathrm{x}} \mathrm{Te}_{4}, \mathrm{Si}_{0.8} \mathrm{Ge}_{0.2}$, and the p-type $\mathrm{Yb}_{14} \mathrm{MnSb}_{11}$ Zintl phase.

\begin{tabular}{lcc}
\hline Material & BOL Sublimation rate $\left(\mathrm{g} / \mathrm{cm}^{2} / \mathrm{hr}\right)$ & Coefficient of Thermal Expansion $\left(10^{-6} / \mathrm{K}\right)$ \\
\hline $\mathrm{Si}_{0.8} \mathrm{Ge}_{0.2}$ & $\sim 8 \times 10^{-5}$ & 5.0 \\
$\mathrm{Si}_{0.8} \mathrm{Ge}_{0.2}$ (Coated) & $\sim 5 \times 10^{-7}$ & 5.0 \\
$\mathrm{Yb}_{14} \mathrm{MnSb}_{11}$ & $\sim 7 \times 10^{-3}(1)$ & $19.9^{(2)}$ \\
$\mathrm{La}_{3-\mathrm{x}} \mathrm{Te}_{4}$ & $\sim 5 \times 10^{-5}$ & 19.4 \\
\hline
\end{tabular}

(1) $\left(\right.$ Paik, 2007); ${ }^{(2)}$ (Ravi, 2007)

\section{CONCLUSIONS}

The key objectives of this work were to improve the synthesis technique of lanthanum telluride, produce samples containing desired stoichiometries with reproducible transport properties, and obtain preliminary data pertaining to key thermal and mechanical properties. The previous synthesis technique was lengthy and susceptible to oxidation and evaporated loss of tellurium, both of which resulted in poor compositional control. To avoid these difficulties, the compound is synthesized at room temperature via a mechanochemical process. Mechanical alloying is utilized to produce a reacted powder at room temperature, which is then hot-pressed to form dense pellets. The technique is fast and allows for many compositions to be studied in a relatively short period of time. With this method, we find TE properties depend on composition in a manner consistent with previous research, and ZT values of approximately 1.0 are achievable at $1275 \mathrm{~K}$ for several $\mathrm{La}_{3-\mathrm{x}} \mathrm{Te}_{4}$ stoichiometries. The beginning of life sublimation rate is approximately $5 \times 10^{-5} \mathrm{~g} / \mathrm{cm}^{2} / \mathrm{hr}$, which compares favorably with state-of-practice $\mathrm{n}$-type $\mathrm{Si}_{0.8} \mathrm{Ge}_{0.2}$. We observed that the coefficient of thermal expansion matched that of another rare earth compound, $\mathrm{Yb}_{14} \mathrm{MnSb}_{11}$, which is currently the best p-type analog for high temperature couple design. Future efforts will focus on (1) the study of the impact of chemical substitutions on thermoelectric properties and (2) the production of large batches of pure lanthanum telluride for detailed investigations of thermal and mechanical stability, as well as potential incorporation into advanced high temperature couples.

\section{ACKNOWLEDGMENTS}

The work described in this paper was performed at the Jet Propulsion Laboratory, California Institute of Technology under contract with the National Aeronautics and Space Administration. The authors would like to thank T. Ikeda for performing the electron microprobe measurements, S. Firdosy and V.A. Ravi for thermal expansion measurements, J. Paik for sublimation rate measurements and L.D. Zoltan and T. McCann for help with TE property measurements. 


\section{REFERENCES}

Brown, S.R., Kauzlarich, S. M., Gascoin, F., and Snyder, G.J., "Yb ${ }_{14} \mathrm{MnSb}_{11}$ : New High Efficiency Thermoelectric Material for Power Generation", Chem. Mater., 18, 7, 1873-1877 (2006).

Caillat, T., Huang, C.-K., Cheng, B., Gogna, P., Firdosy, S., Ravi, V., "Physical and Thermoelectric Properties of Ball Milled $\mathrm{Yb}_{14} \mathrm{MnSb}_{11} "$, Abstract book, of the 2007 International conference on Thermoelectrics, 2007.

Cutler, M., Leavy, J.F. and Fitzpatrick, R.L., "Electronic Transport in Semimetallic Cerium Sulfide", Phys. Rev. 133, 4A, $1143-$ 1152 (1964).

Cutler, M., and Leavy, J.F., "Electronic Transport in High Resistivity Cerium Sulfide", Phys. Rev. 133, 4A, 1153-1162 (1964).

Danielson, L.R., Alexander, M., Vining, C., Lockwood, R.A., C. Wood., C., A., "Thermoelectric properties of LaTe,", Proc. $7^{\text {th }}$ International Conference on Thermoelectrics, edited by K.R. Rao, The University of Texas at Arlington, 1988, pp. 71-75.

Paik, J., Private Communication, 2007

Ramsey, T. H., Steinfink, H., Weiss E. J., "The Phase Equilibria and Crystal Chemistry of the Rare Earth-Group VI Systems. IV. Lanthanum-Tellurium", Inorg. Chem., 4, 1154-1157 (1965).

Ravi, V., Private Communication, 2007

Vining, C., Wood., C., Parker, J., Zoltan, A., Danielson, L.R., Alexander, M.N., "Electrical and Thermal Transport in Lanthanum Telluride", Proc. $7^{\text {th }}$ International Conference on Thermoelectrics, edited by K.R. Rao, The University of Texas at Arlington, 1988, pp. 9-13.

Wood. C., Lockwood, A., Parker, J., Zoltan, A., and Zoltan, D., "Thermoelectric Properties of Lanthanum Sulfide", J. Appl. Phys. 58 (4), 1542-1547 (1985).

Wood. C., "Materials for Thermoelectric Energy Conversion", Rep. Prog. Phys. 51, 459 (1988). 